\title{
INVESTIGACIONES
}

\section{La mentoría en el impacto académico del profesorado novel de la UPV/EHU}

\author{
The mentoring in the academic impact on beginner teachers \\ in the University of the Basque Country
}

\author{
Itziar Rekalde-Rodríguez, Aitor Mendia-Urrutia ${ }^{a^{*}}$ \\ ${ }^{a}$ Universidad del País Vasco/Euskal Herriko Unibertsitatea. \\ itziar.rekalde@ehu.eus, aitor.mendia@ehu.eus
}

\section{RESUMEN}

La Universidad del País Vasco (UPV/EHU), en su afán por ofrecer una formación de calidad ante el relevo generacional de su profesorado, lanzó el programa iRAKER imbricando la mentoría para acompañar al profesorado novel en su desarrollo académico. El objetivo de este trabajo es analizar y comprender el impacto que el programa iRAKER ha tenido sobre el profesorado participante (novel y sénior). El método abordado es el estudio de caso, y los/as participantes 18 docentes noveles y 19 sénior. Los instrumentos utilizados han sido: entrevistas, encuestas, grupo de discusión y análisis documental. Los resultados revelan que el efecto de la mentoría imbricada en un programa formativo impacta, a nivel individual, en la producción científica y, a nivel institucional, en el compromiso y la visibilización social de la universidad. Se concluye sugiriendo pautas de actuación transferibles a otras universidades para aplicar la mentoría en procesos de formación del profesorado universitario.

Palabras claves: formación del profesorado, educación superior, estrategia de enseñanza, competencia académica, seguimiento del profesorado.

\begin{abstract}
The University of the Basque Country (UPV/EHU), in its eagerness to offer quality training in the face of the generational change of its teaching staff, launched the iRAKER program where the mentoring was inserted to accompany the new teaching staff in their academic development. The aim of this work is to analyze and understand the impact that the iRAKER program has had on the participating teachers (novel and senior). The method addressed is the case study, and 18 novel teachers and 19 senior have participated. The instruments used are interviews, inquests, focus group and documental analysis. Findings revealed that the effect of mentoring set in a training program impacts on scientific production, at individual level, and, in social engagement and visibility of the university at institutional level. It is concluded by suggesting transfer guidelines for the development of mentoring in university teacher training processes.
\end{abstract}

Key words: teacher training, higher education, teaching strategy, academic competence, teacher monitoring.

\footnotetext{
* Disfruta actualmente de una beca pre doctoral en la convocatoria de contratación para la formación de personal investigador en la UPV/EHU 2019.
} 


\section{INTRODUCCIÓN}

Las personas que investigan la formación del profesorado entienden que el desarrollo profesional docente es un proceso amplio, flexible, continuo y caracterizado por una reflexión constante sobre la experiencia diaria en el que se incluyen oportunidades y experiencias planificadas que promueven el crecimiento y el desarrollo de la profesión (Boyer, 1990; Paricio, Fernández y Fernández, 2019). Este desarrollo profesional se concibe como un proceso colaborativo que, sin suprimir espacio para el trabajo y la reflexión personal, consigue los mayores beneficios en las interacciones significativas con otros docentes y, por tanto, implica la consideración de las y los profesores como aprendices activos comprometidos en la preparación, observación y reflexión sobre su práctica (Fernández et al., 2013).

La profunda transformación producida en el sistema educativo universitario europeo, ha incidido en el profesorado universitario novel por el modo en que se está socializando profesionalmente y haciendo frente a las problemáticas intrínsecas -docente e investigadora- de la profesión académica (Knight, 2006).

Desde esta panorámica se observa que el colectivo de docentes noveles requiere de una mayor atención por hallarse en muchos casos inseguros, con falta de confianza e intentando establecer su propia identidad profesional y, añadiendo a todo esto, el hacer carrera universitaria. Desde hace décadas han surgido diferentes propuestas para atender la formación de este colectivo (Bozu, 2010; Fernández, 2008; Sánchez y Mayor, 2006), aunque aquellos que insertan la estrategia de la mentoría en sus procesos formativos reconocen que es eficaz para la formación inicial y continua del profesorado universitario (Cullingford, 2016; Laverick, 2016; Sánchez et al., 2015).

La política de la Universidad del País Vasco (UPV/EHU) ha demostrado estar pendiente en todo momento de los cambios sociales y las demandas del personal de su comunidad (Alkorta, 2012; Fernández y Palomares, 2011; Fernández et al., 2013), y un problema con el que se encuentra en la actualidad es el relevo generacional que la comunidad universitaria está viviendo. Al objeto de formar al profesorado novel de la UPV/EHU se puso en marcha el programa iRAKER, que pretende desarrollar la competencia académica del profesorado universitario novel haciendo uso de la mentoría como estrategia de acompañamiento y consolidación de este profesorado docente e investigador (PDI) en la institución universitaria. A través de evaluar intervenciones y prácticas como ésta, se consigue una universidad que aprende de lo que hace, se transforma y transfiere lo aprendido a otros contextos (Arandia y Rekalde, 2014; Fernández y Rekalde, 2011).

\section{LA FORMACIÓN DEL PROFESORADO. HACIA EL DESARROLLO DE LA COMPETENCIA ACADÉMICA}

El Espacio Europeo de Educación Superior propició un contexto lleno de cambios en el que se crearon expectativas, proyecciones y malestares que aceleraron discusiones que la universidad tenía pendiente (Gijón y Crisol, 2012). Entre otros temas cruciales estaba el del profesorado universitario y su formación (Margalef y Álvarez, 2005) en el que la innovación y la formación continua se establecen como indicadores para la calidad y excelencia (Mas, 2011). 
Ante esta situación los organismos universitarios competentes cambian el tipo de formación ofrecida a su profesorado; transitando de una puntual a través de cursos y seminarios, a programas más amplios y complejos (Margalef y Álvarez, 2005). Se dan cuenta que no sólo es necesario que el/la docente sepa de la materia, sino que sea competente en cómo enseñar la disciplina (López, 2011) y, en acompañar al alumnado a lo largo de su proceso de aprendizaje (Lobato y Guerra, 2016).

\subsection{LA FORMACIÓN DEL PROFESORADO NOVEL ¿QUÉ HAY DE NUEVO?}

Los actuales estudios sobre la formación del profesorado novel constatan que:

- los programas de formación del profesorado novel inciden básicamente en la competencia docente desarrollando habilidades vinculadas con la enseñanza (planificación, metodología, evaluación, puesta en escena...), la gestión universitaria (tramitación de proyectos, adecuación de programas...) y, las relaciones interpersonales (con el alumnado y profesorado) (Herrera, Fernández, Caballero y Trujillo, 2011). En este sentido, las investigaciones avalan que una mayor formación en el ámbito de la docencia deriva en el empleo de métodos de enseñanza centrados en el alumnado (Jiménez, González y Tornel, 2019; Gibbs y Coffey, 2004; Postareff, Lindblom-Ylänne y Nevgi, 2008) y en la mayor utilización de diferentes métodos de enseñanza (Light, Calkins, Luna y Drane, 2009; Drew, 2004; Trigwell y Prosser, 2004; Hativa, 2000). El profesorado universitario reivindica:

- un mayor reconocimiento a la labor docente (Díaz, 2018), dado que los actuales criterios de promoción profesional se centran en los méritos en investigación y no tanto en los de la docencia (Coba, 2011, cit en Díaz, 2018).

- una formación que integre en su totalidad los diferentes ámbitos de actuación de la persona académica (Sánchez y Mayor, 2006).

Por todo esto, para el desarrollo de la competencia académica es imprescindible el establecer programas y procesos formativos sistemáticos y de calidad (Marentic, 2009); programas flexibles y adaptados a los contextos; que respondan a las necesidades de los y las participantes (Madinabeitia y Fernández, 2017; Zabalza, 2003) y que operen desde una visión comprensiva (Fernández et al., 2013) para que los beneficios no sólo reviertan en la institución sino en toda la sociedad (ENQA, 2015).

Pero, no sólo consiste en diseñar e implementar la formación, sino también en evaluarla y, esto, no es una tarea fácil, aunque sí necesaria. Según Pineda (2000) sólo evaluando se pueden detectar los efectos de las acciones formativas, los rendimientos de la inversión efectuada y tomar decisiones para optimizar la calidad de la formación futura.

El modelo más extendido para evaluar los resultados de un programa formativo sigue siendo el clásico de Kirkpatrick y Kirkpatrick (2000) que establece cuatro niveles: (1) satisfacción o reacción, como el grado de satisfacción que los y las participantes tienen con la formación (normalmente a través de cuestionarios); (2) aprendizaje (o desarrollo de competencias), que significa evaluar el grado de adquisición de conocimientos y/o desarrollo de competencias de los y las participantes; (3) transferencia, en relación a la aplicación de lo aprendido y (4) impacto, que evalúa la repercusión de la formación a nivel individual, 
colectivo u organizativo. En cuanto a la transferencia, el objetivo es lograr el impacto en el conjunto de la organización y/o en los usuarios últimos, por lo que, en caso de no poder evaluar todo ello, se pueden centrar los esfuerzos en evaluar lo más relevante (Cano, 2016).

\subsection{LA MENTORÍA EN LA FORMACIÓN DEL PROFESORADO NOVEL. AHONDANDO EN SU UTILIZACIÓN}

La formación inicial del profesorado universitario es uno de los avances más importantes para la mejora de la calidad de la docencia en las instituciones de Educación Superior, y esta formación se asienta en la idea básica de que el mejor modo de responder a los desafíos planteados desde la sociedad del conocimiento y del aprendizaje a lo largo de la vida, es la profesionalización de la docencia universitaria (Fernández, 2008; Paricio, Fernández y Fernández, 2019). Si bien el profesorado novel está bien formado en cuanto a su ámbito de conocimiento, no lo está para la transferencia de éste al alumnado (Fernández, 2003; Herrera, Fernández, Caballero y Trujillo, 2011; Sánchez y Mayor, 2006).

La formación del profesorado novel es la que se desarrolla al inicio de su carrera académica, y es clave para que se adapte y socialice en la cultura institucional al tiempo que influye en su identidad profesional futura (Bozu, 2010; Eirín, García y Montero, 2009; Herreras, 2007). Los programas formativos dirigidos al profesorado novel deben cumplir las siguientes condiciones: 1) aportar apoyo emocional; 2) estar vinculados a la realidad; 3) demostrar compromiso por parte del profesorado novel; 4) ofrecer apoyo a lo largo del tiempo; 5) tener una visión de futuro, dado que lo que se lleva a cabo al inicio influye en el futuro de los y las docentes, y 6 ) tener en cuenta que las políticas institucionales tienen un impacto directo en el bienestar del profesorado novel (Bozu, 2010).

Una estrategia formativa eficaz para la formación inicial y continua del profesorado universitario que tiene en cuenta los aspectos señalados es la mentoría (Cullingford, 2016; Laverick, 2016; Sánchez et al., 2015), en el que un profesor/a de reconocida experiencia profesional (mentor/a) asesora y apoya a otro/a de menor recorrido (mentorizado/a) (Ballesteros y Nubiola, 2013). Este encuentro es, en la mayoría de los casos, fortuito en la universidad (Rekalde y Guerra, 2018) y sólo en algunos provocado y sistemático. Por esto, González, Araneda, Hernández y Lorca (2005) señalan que la transmisión no será totalmente valiosa sino se establece de manera sistemática y formal. Por su parte, Smith e Ingersoll (2004) subrayan que el profesorado novel a menudo se encuentra solo y perdido en su puesto de trabajo, y para evitarlo el apoyo de una persona experimentada es crucial. Los estudios apuntan a que es una estrategia beneficiosa para ambos y, para la propia institución (Eirín, García y Montero, 2009; Vonk, 1996), para integrar al novel en la cultura de la institución universitaria (Anafarta y Apaydin, 2016; Allen y Eby, 2010), aumentar su compromiso con la organización (Falzarano y Zipp, 2012; Allen et al., 2009), favorecer su permanencia (Bergevin y Martineau, 2007; Falzarano y Zipp, 2012), incrementar su confianza y sentimiento de eficacia en sus tareas (Anafarta y Apaydin, 2016; Fletcher y Mullen, 2012; Harvey et al., 2017), aprender de manera autónoma y colaborativa (Hobson et al., 2009), tener una mayor satisfacción profesional (St-Jean y Audet, 2013) y, desarrollar las competencias académicas del profesorado universitario (Harvey et al., 2017; Herrera, Fernández, Caballero y Trujillo, 2011).

La persona mentora en este proceso es clave y, según Vélaz de Medrano (2009), debe ser un/a profesional competente que asesora y orienta las diferentes dimensiones del 
aprendizaje y desarrollo de la carrera profesional académica. No obstante, la literatura ha revelado que existe un vacío en la investigación acerca de la sinergia entre las funciones de ambos agentes y cómo estas funciones interactúan y reaccionan (Andreucci, 2018). Cherian (2007), por su parte, indica que las funciones que se espera que cumplan las personas mentoras y la forma como las realizan no están bien documentadas en la investigación actual.

Además, Smith e Ingersoll (2004) señalan que no todos los programas de mentorización son iguales y que es importante la duración del programa y si el profesorado mentor y, los y las mentorados/as han recibido formación. Según Fernández, González y Belando (2017) para que los programas de mentoría sean exitosos deben: 1) propiciar relaciones de confianza entre los y las participantes; 2) aprender de los y las iguales como objetivo; 3) proponer mentores/as competentes a diferentes niveles (cognitivo, investigador, tecnológicopedagógico, interpersonal, meta cognitivo y ético); 4) impulsar el diálogo y crear contextos interactivos, colaborativos, democráticos e informales, y 5) construir puentes entre la formación inicial y la realidad laboral. Smith e Ingersoll (2004) concluyen, además, que si el mentor/a y el o la novel son de la misma área de conocimiento, hay más probabilidad de que el proceso de mentoría sea exitoso. Al igual que si comparten características personales (Hobson et al., 2009; Rippon y Martin, 2006). Teniendo en cuenta todo lo anterior, el presente trabajo tiene por finalidad el desvelar el impacto que la utilización de la mentoría como estrategia de seguimiento y acompañamiento en el desarrollo de la competencia académica tiene sobre el profesorado participante en iRAKER (programa formativo para el profesorado novel de la UPV/EHU), para, a la luz de esta exploración, dibujar pautas de actuación transferibles a otras universidades. Los objetivos son:

- Describir la valoración que el profesorado experimentado y novel hace del proceso de mentorización imbricado en el Programa formativo iRAKER para el desarrollo de la competencia académica.

- Analizar la repercusión que la mentoría, inserta en el programa formativo iRAKER, ha tenido en el desarrollo docente, investigador y de gestión del profesorado novel a nivel individual e institucional.

\section{MÉTODO}

\subsection{ENFOQUE METODOLÓGICO}

En coherencia con el marco teórico y los objetivos del estudio, se ha optado por un enfoque interpretativo al ser idóneo para analizar percepciones, valoraciones y reflexiones respecto a las vivencias de las personas investigadas para que quien investiga comprenda sus puntos de vista (Janesick, 2000). Este enfoque se ha articulado de manera cualitativa (Taylor y Bogdan, 1996) al objeto de penetrar y comprender las valoraciones vertidas por los y las participantes sobre la mentoría, para contribuir a dibujar pautas de actuación transferibles a otras universidades (Grundy, 1991; Flick, 2011).

La manera de abordar esta investigación ha sido a través del estudio de caso. Diferentes autores señalan que constituye una de las estrategias más características del enfoque cualitativo (Stake, 1998; Vázquez y Angulo, 2003), y lo encuentran particularmente 
apropiado para estudiar un caso o una situación con intensidad (Stake, 1998). Esta definición nos remite a la indagación de un fenómeno con la intención de comprenderlo y a una unidad de estudio que puede ser un individuo, un marco, una organización o, como en este caso, la mentoría como estrategia formativa imbricada en el programa iRAKER para la formación de la competencia académica del profesorado universitario novel.

\subsection{PARTICIPANTES}

Las personas participantes en esta experiencia son, por una parte, el profesorado novel participante en la primera edición del programa iRAKER 2017/2018 que fueron 18 docentes, de ellos 7 hombres y 11 mujeres y, por otra, el profesorado mentor que han sido 19 , de los cuales 11 son hombres y 8 mujeres.

El perfil de los y las noveles es el siguiente: la edad promedio es de 36 años, 6 docentes pertenecen al área de Ciencias de la Salud, 4 Ciencias Sociales y Jurídicas, 4 Ciencias Técnicas, 3 Ciencias experimentales y, 1 Artes y Humanidades. Los años de experiencia del profesorado muestran que todos/as excepto 1 lleva más de 5 años en la docencia universitaria.

El profesorado experimentado participante ha sido de 19 docentes de diferentes departamentos y áreas de conocimiento: 3 de Ciencias Sociales y Jurídicas, 1 de Artes y Humanidades, 7 de Ciencias de la Salud, 4 de Ciencias Técnicas y 4 de Ciencias Experimentales. Todo el profesorado mentor disfruta de, al menos, 2 sexenios de investigación, evaluación positiva del programa de evaluación docente Docentiaz y, formación por parte del Servicio de Asesoramiento Educativo de la UPV/EHU (SAE/ HELAZ).

\subsection{INSTRUMENTOS DE OBTENCIÓN DE INFORMACIÓN}

Los instrumentos de investigación utilizados han sido los siguientes:

1) Entrevistas en profundidad. Como señalan Jordi y Macías (2014) se ha tenido presente que, al entrevistar, el saber escuchar es tanto o más importante que el saber preguntar. Partiendo de este supuesto, la entrevista en profundidad ha facilitado: una implicación interpersonal con el entrevistado, una aclaración de cuestiones o dudas que han surgido a lo largo de la entrevista, y una observación gestual de lo que acontecía que se ha ido registrando rigurosamente (Lukas y Santiago, 2009). Las preguntas de las entrevistas están inspiradas en el instrumento creado por Feixas et al. (2013) para medir la transferencia de un programa de formación. Las entrevistas se han llevado a cabo un año después de terminar con el proceso de mentoría al objeto de comprobar la valoración que los y las noveles hacen del impacto de la formación al cabo del tiempo (Madriz, 2008).

2) Encuesta descriptiva de satisfacción. La utilización de este instrumento es adecuada para obtener información de manera ágil y rápida, respetando el anonimato de los y las participantes (Casas, Repullo y Donado, 2003). En este estudio se ha utilizado para conocer la tendencia de las valoraciones que los y las participantes realizan respecto a la estrategia de la mentoría imbricada en la formación y no con el propósito de cuantificar las valoraciones ni establecer relaciones causales entre las variables. La encuesta del profesorado novel es 
una escala numérica que consta de 26 preguntas cerradas agrupadas en 5 bloques temáticos y 6 preguntas abiertas. Respecto a la escala del profesorado experimentado se recogen 12 preguntas cerradas y 6 abiertas. Todas las encuestas se han contestado online.

3) Grupo de discusión. Este instrumento promueve la participación e interacción grupal, haciendo que el profesorado novel construya información en el propio escenario grupal donde, estimulado por la presencia de compañeros/as emite opiniones, y se obtiene información de primera mano (Callejo, 2001; Infesta, Vicente y Cohen, 2012). Las preguntas focales que dirigieron la discusión en el grupo de profesorado novel apuntaban a visualizar la percepción que tenían sobre el desarrollo de la competencia académica y el trabajo a desarrollar con su mentor/a.

4) ePortfolios. Se ha llevado a cabo un análisis de los eportfolios de los y las noveles centrando el foco en el proceso de la mentoría. En esta formación los ePortfolios han constituido instrumentos formativos en los que se ha ido recogiendo la síntesis del efecto que la formación tiene en el desarrollo académico del profesorado novel a lo largo de los 18 meses.

Si bien el ePortafolio académico se utiliza como nexo de unión de los aprendizajes obtenidos y para evidenciar el desarrollo de competencias (Collins, Hawkins, y Fredriksen, 1991; Ojeda, 2007; Palomares, 2011; Shulman, 1999) es, también, un instrumento de obtención de información muy interesante en la investigación educativa (Cebrián, 2011; Orbea, Cruz y Rekalde, 2019).

\subsection{EL CONTEXTO DE INVESTIGACIÓN Y EL TRABAJO DE CAMPO}

Esta investigación se ha desarrollado en los contextos próximos en los que interviene el profesorado; desde el nivel macro como institución universitaria cuya política formativa influye en el desarrollo de la competencia académica del profesorado, hasta el nivel meso equivalente al programa formativo iRAKER ofrecido por el SAE/HELAZ, llegando al nivel micro en el que se analiza el impacto de la mentoría en el profesorado participante en el programa iRAKER.

El proceso formativo en su totalidad se recoge en la figura 1, en la que se diferencian tres módulos de formación a lo largo de 18 meses en el que la estrategia de la mentoría se inserta en el segundo y tercer módulo. Es a lo largo del primero donde se diseña, por parte del profesorado novel y las personas formadoras del SAE/HELAZ, el Plan de Aprendizaje Profesional (PAP) individualizado en el que se recogen metas de aprendizaje para cada uno de los ámbitos que la literatura recoge claves para el desarrollo de la competencia académica (docencia, investigación y gestión). Este módulo es clave porque la construcción del PAP, ayuda en la designación de las personas mentoras y sirve como carta de navegación orientativa del proceso formativo para ambos.

El trabajo de campo se ha llevado a cabo a lo largo de la formación utilizando instrumentos de obtención de datos construidos expresamente para la investigación (grupo de discusión -cierre módulo 1-, encuesta descriptiva de satisfacción -cierre módulo 2-, entrevistas en profundidad -un año después de finalizar la formación-). Así como actividades formativas que han sido objeto de análisis para nutrir la investigación (ePortfolio -elaborado a lo largo de la formación-). 
Figura 1. Programa formativo iRAKER

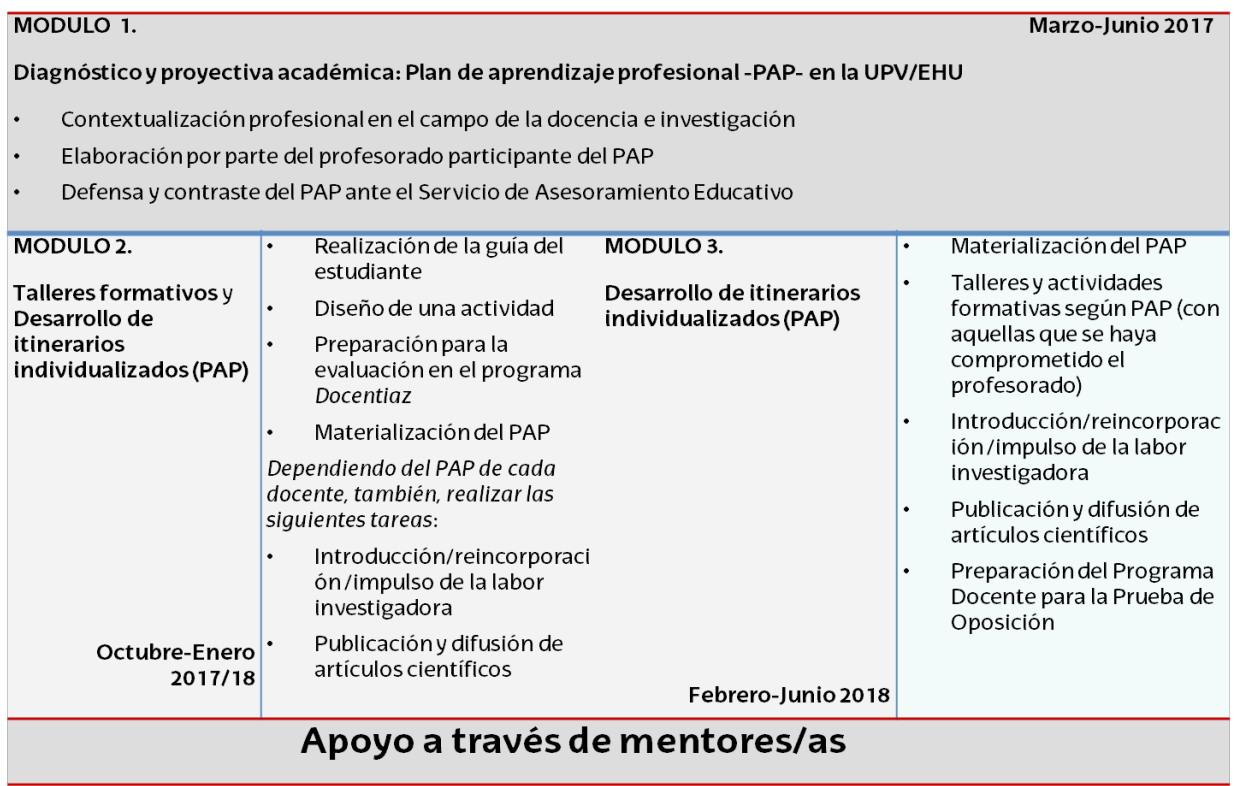

Fuente. Elaboración propia.

\subsection{ANÁLISIS DE DATOS Y CATEGORÍAS DE ANÁLISIS}

Los datos de naturaleza cuantitativa, que provienen de la escala numérica construida para indagar en la satisfacción de los participantes respecto a la estrategia de la mentoría, se han analizado respetando el anonimato y, por lo tanto, reduciendo la influencia de las personas investigadoras sobre los y las participantes (Casas, Repullo y Donado, 2003).

Los datos cualitativos, que provienen de las entrevistas, el grupo de discusión, preguntas abiertas de la encuesta y ePortfolio fueron sometidos a un proceso recursivo de análisis inductivo (Braun y Clarke, 2006; Patton, 2002) para establecer temas recurrentes (Flick, 2011; Patton, 2002) y obtener como resultado un sistema categorial flexible y abierto en la línea que apunta Cabrera (2000, cit. en Lukas y Santiago, 2009) (véase tabla 1). 
Tabla 1. Sistema categorial



Fuente. Elaboración propia.

\subsection{CALIDAD DE LOS DATOS}

El criterio por excelencia para la investigación naturalista es la veracidad que se articula y se plasma en este trabajo a través de las siguientes características de la triangulación múltiple (Aguilar y Barroso, 2015; Araneda, 2006; Beltrián et al., 2013): 1) credibilidad; se ha intentado recopilar toda la información posible con la pretensión de reconstruir el impacto que el proceso de mentoría ha tenido sobre los y las participantes (Tójar, 2001). De hecho, desde el primer momento el profesorado ha formado parte del fenómeno que 
se trata de comprender para garantizar que se aborda lo que ellos y ellas consideran clave/ problemático y propiciar su cambio de mirada, 2) transferibilidad; se pretende transferir lo realizado en este estudio a otros contextos similares porque, en cierto sentido, el profesorado universitario está inmerso en una situación similar de constante acreditación (formación, evaluación...), 3) dependencia; se han aplicado diferentes técnicas que han permitido obtener resultados muy similares, lo que permite deducir el carácter de estabilidad de los mismos y, 4) confirmabilidad; se subraya al utilizar descriptores de bajo nivel de inferencia (transcripciones y documentos de fuentes primarias) registrando lo más fielmente posible la realidad.

\subsection{FACTORES ÉTICOS}

En sintonía con lo que proponen Blaxter, Hughes y Tight (2008), a las personas participantes se les aclaró desde el principio la naturaleza del estudio y en qué consistía el consentimiento informado. Se llegó a acuerdos sobre el uso de los datos, su análisis y divulgación. Las voces de las personas participantes han sido codificadas siendo un elemento más para preservar la privacidad y el anonimato, y los valores como la dignidad e integridad (Arregi et al., 2008). Así mismo, el respeto por la libertad y autonomía de los y las participantes se ha defendido en todo momento, tal y como indica el Comité de Ética para las Investigaciones relacionadas con Seres Humanos de la UPV/EHU (CEISH, 2019), y se ha cuidado el tratamiento del género a lo largo del trabajo.

\section{RESULTADOS}

Seis son las dimensiones a través de las cuales se han articulado los resultados de este estudio: 1) valoración del proceso de designación de las personas mentoras; 2) relación y clima de trabajo entre el PDI novel y experimentado; 3) beneficios y obstáculos en el proceso de mentoría, 4) satisfacción de los y las participantes con la estrategia de la mentoría inserta en el programa de formación, 5) valoración de la mentoría sobre la competencia académica en los y las noveles y, finalmente, 6) efecto de la mentoría en términos de producción científica.

\subsection{PROCESO DE DESIGNACIÓN DE PERSONAS MENTORAS: DIÁLOGO Y CONSENSO ENTRE LAS PARTES IMPLICADAS}

El proceso global de designación de los y las mentoras se valora por el profesorado novel con un 4,9 sobre 5, dimensión en la que se valora que se sienten muy conformes respecto a la designación de sus mentores y mentoras $(4,9)$ : "Estoy muy contenta con la mentora asignada, aunque no sea de mi campo de conocimiento. Estoy encantada de haberla conocido" (PDI-novel).

Así también, se ha valorado el procedimiento de designación seguido $(4,9)$ que ha consistido en un diálogo a tres bandas entre la propuesta inicial de mentor/a del novel, el contraste de ésta con el servicio-institución (SAE/HELAZ) y, finalmente la aceptación de la asunción del rol de mentor/a por parte del PDI experimentado: "Me ha parecido perfecto que se tuviera en cuenta desde el principio las/los mentores citados a modo de sugerencia por nosotros" (PDI-novel). 
Es de subrayar que muchos de los y las participantes comentan que su propuesta inicial de mentor/a se ha debido a que esta persona venía ejerciendo, de manera informal, como tal. Ha sido una persona que, desde que están en la universidad, les ha ayudado siempre que lo han necesitado. Reconocen que el nombrarles de manera formal mentores/as le concede valor y reconocimiento institucional al trabajo que llevan a cabo: "No hay un protocolo de acogida, cuando entra un docente no sabe lo que hacer, y es muy interesante la figura del mentor" (PDI-novel).

\subsection{RELACIÓN Y CLIMA DE TRABAJO: COMPLICIDAD PAUTADA}

Se valora en su conjunto por el profesorado novel la relación establecida con el profesorado experimentado y el clima de trabajo creado con un 4,76 en el que el profesorado novel valora que la frecuencia con la que se ha reunido con su mentor/a es adecuada y pertinente $(4,6)$, que el tiempo invertido en las reuniones es suficiente $(4,8)$, que las reuniones le han ayudado a indagar y reflexionar sobre su proceso formativo $(4,7)$, que ha sido fácil acerarse a la persona mentora y hablar con él/ella $(4,8)$ y, que ha recibido feed-back de manera continua por parte de su mentor/a $(4,8)$ :

Tengo a una persona con la que puedo hablar libremente. A veces es difícil hablar sobre determinados temas con el resto de compañeros, y el tenerle al lado hace que cualquier duda pueda ser dilucidada. Además, tiene una disposición plena. Está completamente comprometido con el proceso de mentoría y siempre tiene la puerta de su despacho abierta, aun siendo una persona cargadísima de responsabilidades (PDI-novel).

Además, el profesorado novel siente que a su mentor/a le interesa su desarrollo académico (4,9): "Entre noviembre y diciembre el equipo docente-investigador ha empezado a carburar, y ya lo veo todo mucho más claro, pero antes de esto, ya hablé con mi mentor docente sobre mi falta de dedicación con la competencia 2 de investigación, y hemos ido resolviendo esos roces de manera satisfactoria" (PDI-novel).

Según el profesorado experimentado considera que la frecuencia con la que se reúne con el/la novel es adecuada y pertinente $(4,5)$, que el tiempo invertido en las reuniones es suficiente $(4,6)$, que en las reuniones tiene presente el itinerario diseñado por el PDI novel para su desarrollo académico $(4,8)$ y, además, que entiende mejor ahora los retos a los que se enfrenta el PDI novel en la UPV/EHU $(4,6)$ : "La experiencia de compartir un trabajo conjunto y muy cercano con una profesora joven, y haber establecido una relación sinérgica y de confianza mutua, que sin el marco de este programa quizás no hubiese sido posible" (PDI- experimentado).

\subsection{BENEFICIOS Y OBSTÁCULOS DEL PROCESO DE MENTORÍA: CONSTANTES EN LA FORMACIÓN}

Los beneficios de la mentoría en su conjunto se valoran por el profesorado novel con un 4,3 en donde se enfatiza que este proceso les está ayudando a planificar mejor su carrera académica $(4,8)$, a crear y/o ampliar su red de contactos (4), a poder tener acceso a recursos (4), a descubrir nuevas oportunidades para su desarrollo académico (4,5), a descubrir nuevas perspectivas en la labor del PDI (4), a visibilizar mejor su carrera académica $(4,6)$, 
a reflexionar en torno a sus tareas como PDI $(4,8)$, a tener más confianza en sí mismo/a $(3,8)$ y a ayudarles en su desarrollo personal $(4,5)$.

En cuanto al PDI experimentado subraya la oportunidad que el programa formativo les ofrece para visibilizar y dar valor a su rol como mentores/as informales en la comunidad universitaria: "Aunque de alguna manera esta labor ya la realizábamos sin tanto compromiso, este programa nos ayuda a reflexionar y a ser mucho más profesionales en nuestras tareas docentes y de investigación" (PDI- experimentado).

Son varios los obstáculos que, de manera reiterada, se repiten en las voces de los y las participantes. Éstos son los siguientes:

- El tiempo. La falta de tiempo es una variable recurrente tanto por el profesorado novel como por el experimentado: "Tiempo, sin duda... un poco de aire para reflexionar, recapacitar y plantear las tareas que tengo por delante..." (PDI-novel).

- Ocasionar molestias. El profesorado novel ha sentido, en ocasiones, que podía estar molestando a su mentor/a al solicitarle ayuda o atención: "Por mi parte, el pedir atención o tiempo a personas que a su vez veo muy atareadas y entender cuál es el papel del mentor/a" (PDI-novel).

- Compaginar tareas. El profesorado experimentado, en cambio, subraya el compaginar diferentes tareas académicas con las funciones propias de mentor/a como un obstáculo recurrente: "Compaginar esta tarea con mis otras múltiples obligaciones: cargos académicos, investigación, dirección de tesis, etc.” (PDIexperimentado).

- Pautas en la mentoría. El seguir un modelo de mentoría, cuando uno/a ya tiene sus modos de hacer es también otra dificultad con la que se han encontrado los y las participantes: "Hacerme a la idea de tener que estructurar la mentoría a los esquemas del SAE, que tampoco ha resultado algo complicado o negativo" (PDIexperimentado).

\subsection{SATISFACCIÓN CON LA MENTORÍA INSERTA EN EL PROGRAMA DE FORMACIÓN: ACOMPAÑAMIENTO Y ACREDITACIÓN}

La satisfacción general del profesorado novel es valorada con un 4,75. Éste subraya su satisfacción con el proceso de acompañamiento que la persona mentora le ha ofrecido $(4,7)$ y con la formación del programa iRAKER $(4,8)$. Según el profesorado experimentado se siente satisfecho/a como PDI al ofrecer apoyo y orientación a otro PDI (5), al contribuir al desarrollo profesional de un PDI novel $(4,9)$ al asumir el rol de mentor/a $(4,5)$ y satisfecho/a con el propio programa formativo $(4,6)$.

El profesorado novel subraya su satisfacción por haber tenido la posibilidad de saber qué es ser académico y dibujar el itinerario a través del cual desarrollar la competencia académica. Se deja entrever la comprensión en su totalidad de la profesión como un factor que les induce a sentir satisfacción: "La verdad es que si miro hacia atrás, el cursar estos módulos ha supuesto conocer y dar respuesta a un mar de dudas, además de aprender un montón. Ha sido el recabar conocimiento para poder tener un buen punto de partida de lo que quiero ser" (PDI-novel).

El profesorado experimentado subraya el apoyo y la ayuda que sienten tener al respaldar desde la institución su labor formativa como mentores/as: "Pienso que el marco 
creado es apropiado y en él es posible trabajar con comodidad, sabiendo también que en caso de dudas existen mecanismos rápidos de solución" (PDI- experimentado).

\subsection{VALORACIÓN DE LA MENTORIA EN EL DESARROLLO DE LA COMPETENCIA ACADÉMICA: LA CLAVE DEL PROFESORADO NOVEL UNIVERSITARIO}

Se valora en su conjunto por el profesorado novel el desarrollo de la competencia académica con un 4,1 en donde el profesorado novel ha valorado que su mentor/a le haya orientado y animado respecto a las metas de aprendizaje competencial incluidas en su PAP $(4,7)$, y respecto a las acciones a desarrollar para conseguir el nivel competencial incluido en el PAP $(4,6)$. En cuanto a los ámbitos que conforman la competencia académica, el relativo al de la docencia valoran que su nivel competencial ha mejorado significativamente (4), en cuanto al de investigación lo valoran con un $(3,8)$, el relativo a gestión universitaria con $(3,5)$, y la responsabilidad social es de $(3,9)$. Según el profesorado experimentado considera que el itinerario diseñado por el/la novel es clave para que mejore su competencia académica $(4,5)$, y según éste le resulta fácil llevar a cabo la tarea del acompañamiento de un PDI $(4,8)$, siente que esta labor le ayuda a indagar y reflexionar sobre su labor como PDI $(4,8)$, y siente que está desarrollando habilidades en orientación académica, y reflexión sobre la práctica académica $(4,8)$.

El ámbito en el que consideran que más han mejorado es el de la docencia: "He estado en permanente contacto con mi mentora, de hecho trabajamos juntas en una de las asignaturas de este cuatrimestre y su ayuda ha sido muy estimulante, me plantea retos, proyectos...es estresante...y apasionante, estoy más que satisfecha con la mentoría" (PDInovel).

No obstante, los y las participantes comentan que la persona mentora cubre aquellos ámbitos en los que el programa incide más tangencialmente como es el de la investigación, y esto lo subrayan muy positivamente: "La parte de la investigación lo he trabajado más con mi mentor que en las sesiones del programa" (PDI-novel).

En cuanto a la gestión y la responsabilidad social, son ámbitos que quedan relegados a un segundo plano: "La mentoría en el programa iRAKER es una gran idea, pero hay problemas de funcionamiento universitario que entorpece y hace que no se puedan desarrollar todas las competencias" (PDI- experimentado).

\subsection{EFECTO DE LA MENTORIA EN EL PROFESORADO NOVEL: FORMACIÓN, PRODUCCIÓN CIENTÍFICA Y VISIBILIZACIÓN SOCIAL Y ACADÉMICA}

La mentoría en el programa iRAKER ha impulsado el desarrollo de la competencia académica tanto en el ámbito de la docencia, investigación, gestión, como en el del compromiso y responsabilidad social. Los datos arrojan los siguientes resultados en términos de formación recibida, artículos publicados, actividades de difusión desplegadas, responsabilidades asumidas por el profesorado novel participante.

Respecto al ámbito de la docencia, la mentoría ha impulsado y facilitado para el desarrollo y la mejora de los procesos de enseñanza-aprendizaje que el profesorado novel se forme en una diversidad de cursos, talleres, seminarios que el SAE/HELAZ ha ofertado y a los que el PDI novel ha acudido y desarrollado satisfactoriamente según información del propio servicio. Temas didácticos como, por ejemplo, planificación docente, evaluación, 
tutoría del alumnado, etc.; temas más instrumentales vinculados a la docencia, investigación o cualquier otro ámbito del desarrollo académico, como uso de programas informáticos o temas vinculados al desarrollo de competencias transversales y de tipo personal del PDI novel como, por ejemplo, temas de género o sostenibilidad.

Así mismo, el profesorado novel se ha formado en cursos y talleres diseñados, desarrollados y evaluados por otros servicios o entidades de la UPV/EHU, como por ejemplo formación desarrollada en centros (facultades e institutos universitarios), en los servicios centrales de investigación, o en la oferta de idiomas que el vicerrectorado de plurilingüismo desarrolla. Es interesante resaltar que, en el transcurso de estos 18 meses de formación, 3 de los PDI noveles se han acreditado como agregados/as o titulares de universidad, y que 6 de ellos/as han obtenido una nota media de Sobresaliente en el programa Docentiaz. Además, cinco de los PDI noveles están participando en Proyectos de Innovación Educativa (PIE) y dos de ellas son coordinadoras de proyecto.

También es de destacar la Movilidad a través de Erasmus + teaching mobility a la cual se han acogido para impartir docencia en otras universidades europeas, o los materiales docentes elaborados dentro del programa Open Course Ware al amparo del Massachusetts Institute of Technology.

Los congresos sobre docencia universitaria en los que el PDI novel ha participado son, entre otros: XIV Foro Internacional sobre evaluación de la calidad de la investigación y la Educación Superior (FECIES), ICERI 2018 y 2019 International Conference on Education, Research and Innovation, INTED 2018, Edulearn-18 y 26 Congreso Universitario de Innovación Educativa en las Enseñanzas Técnicas.

Para resumir, los resultados en el ámbito de la docencia arrojan 1727 horas de formación impartidas por el SAE/HELAZ, que hace un promedio de 96 horas, aproximadamente, de formación por novel. Complementada con 212 horas formativas impartidas por otros servicios e instituciones de la UPV/EHU, y con 107 horas más de formación externa. Así mismo, un resultado significativo es la iniciación de los y las noveles en la innovación docente a través de PIEs, así como en la publicación y difusión de experiencias sobre procesos de enseñanza-aprendizaje en el aula a través de los congresos de innovación educativa.

Respecto al ámbito de la investigación, los datos recogidos en términos de producción científica nos arrojan los siguientes resultados (véase tabla 2): 
Tabla 2. Número de artículos publicados en revistas científicas según el campo de conocimiento

\begin{tabular}{|l|r|r|r|}
\hline Ciencias Sociales y Jurídicas & \multicolumn{2}{c|}{ Artes y Humanidades } \\
\hline Revistas & $\mathbf{N}^{\circ}$ artículos & Revistas $\mathbf{N}^{\circ}$ de artículos \\
\hline JCR & 7 & JCR \\
\hline Scopus & & Scopus & 1 \\
\hline Otras & 6 & Otras & 1 \\
\hline
\end{tabular}

\begin{tabular}{|l|l|l|l|l|l|l|}
\multicolumn{2}{c|}{ Ciencias de la Salud } & \multicolumn{3}{c|}{ Ciencias Experimentales } & Ingeniería y Arquitect ura \\
\hline Revistas & $N^{\circ}$ artículos & Revistas & N $^{\circ}$ artículos & Revistas & $N^{\circ}$ artículos \\
\hline Q1 & 3 & Q1 & 5 & Q1 & 11 \\
\hline Q2 & 2 & Q2 & 1 & Q2 & 6 \\
\hline Q3 & & Q3 & & Q3 & 1 \\
\hline Q4 & 2 & Q4 & & Q4 & \\
\hline
\end{tabular}

Fuente. Elaboración propia.

El profesorado novel reconoce el apoyo y la guía por parte de las personas mentoras en la producción investigadora. Se han agrupado los artículos en función de los campos de conocimiento de la ciencia en los que se ha definido el perfil del profesorado novel: 29 artículos publicados, de los cuales 13 de Ciencias Sociales y Jurídicas, 2 de Artes y Humanidades, 7 de Ciencias de la Salud, 6 de Ciencias Experimentales y 18 de Ingeniería.

En Ciencias Sociales y Jurídicas de los 7 artículos publicados por el JCR, 2 de ellos son Q1 y 5 Q3, en Ciencias de la Salud 3 de los 7 artículos están en revistas Q1, en Ciencias Experimentales 5 de los 6 están en Q1 y en Ingeniería 11 de los 18 están en el primer cuartil.

Durante el programa han publicado, así mismo, 4 capítulos de libro y han participado en congresos por todo el mundo según su campo de conocimiento con 74 trabajos aportados (véase tabla 3).

Tabla 3. Número de aportaciones a congresos científicos según el campo de conocimiento

\begin{tabular}{|c|c|}
\hline Congresos & $\mathbf{N}^{\circ}$ aport aciones \\
\hline Ciencias Socialesy Jurídicas & 18 \\
\hline Artes y Humanidades & 2 \\
\hline Ciencias de la Salud & 17 \\
\hline Ciencias Experimentales & 15 \\
\hline Ingeniería y Arquit ectura & 22 \\
\hline
\end{tabular}

Fuente. Elaboración propia. 
Estamos hablando de 107 producciones científicas en los diferentes campos de conocimiento que gracias al apoyo, guía y acompañamiento de las personas mentoras han podido visibilizarse e impactar en los respectivos campos del conocimiento.

Respecto a la participación y el compromiso adquirido por los y las noveles en el ámbito de la gestión, los resultados destacan que la mitad del PDI novel ha asumido la coordinación de alguna de las asignaturas que imparte y que de esos 9 noveles 6 de ellos/ as son las personas coordinadoras, además, de una segunda asignatura. Todos ellos y ellas llevan la dirección de TFGs y es significativo que justo al finalizar la formación una de las participantes haya asumido la Dirección de un Departamento y, a otra se le haya nombrado Directora de Relaciones Externas del Campus de Bizkaia. Seis de las personas participantes han asumido la organización de congresos, jornadas, seminarios, etc.; vinculados a su campo de conocimiento como tarea de gestión y organización vinculada al departamento y área.

En cuanto al ámbito del compromiso y la responsabilidad social, los resultados subrayan, la participación de los y las noveles en actividades de difusión de la ciencia que impulsan, por ejemplo, entidades reconocidas en el contexto cultural vasco, como son Elhuyar Fundazioa y Eureka Zientzia Museoa.

\section{DISCUSIÓN Y CONCLUSIONES}

La valoración que el profesorado experimentado y novel hace del proceso de mentorización imbricado en el Programa formativo iRAKER para el desarrollo de la competencia académica del novel, pone de relieve los siguientes aspectos:

- Siguiendo la tesis de Vonk (1996), en esta investigación se confirma que el proceso de mentoría es un éxito en gran medida por la cercanía académica de la persona mentora respecto a los y las participantes. Y, como apuntan diferentes autores/as, existe mayor probabilidad de éxito si ambos son del mismo campo de conocimiento (Smith e Ingersoll, 2004). No obstante, el proceso de designación ha sido largo y complicado, porque no sólo exige la identificación de las personas mentoras (Fernández, González y Belando, 2017; Velaz de Medrano, 2009), sino la compatibilidad entre ambas y ha habido casos en los que asignar un mentor/a externo al contexto del participante ha sido oxigenante para él/ella. Se puede concluir diciendo que el éxito en iRAKER se debe al procedimiento utilizado para llegar a la elección de la persona mentora. Un proceso de diálogo y negociación entre las partes implicadas: mentorado/a, persona mentora e institución.

- La relación establecida a través de la mentorización ha impactado en los y las participantes porque ha sido sistemática, basada en la continuidad con la persona mentora y con la intención de ofrecerle respaldo e información (González, Araneda, Hernández y Lorca, 2005).

- Se hace hincapié en la confianza creada para preguntar, comentar, discutir cuestiones relativas al desarrollo académico, a la institución; la asunción del rol de mentor/a propicia esta complicidad pautada (Fletcher y Mullen, 2012; Anafarta y Apaydin, 2016). No obstante, los y las noveles reconocen que ha sido difícil, en ocasiones, pedir ayuda a personas con tantas responsabilidades y tareas (Lucas, 
2001), porque ello les ha generado la sensación y el sentimiento de molestar.

- Se subraya el hecho de que la mentoría ha beneficiado al profesorado novel a planificar mejor su carrera académica, a ser más consciente y diseñar el itinerario por el que transitar para desarrollar las tareas propias de la persona académica en los diferentes ámbitos de actuación (Harvey et al., 2017), pero también a reflexionar sobre sus tareas como PDI (Allen et al., 2009).

- Se incide en la necesidad e idoneidad de reconocer a quienes ejercen de manera informal como mentores/as en la universidad y asignarles un rol visible y reconocido por la institución (González, Araneda Hernández y Lorca, 2005), cuestión ésta, la del status, la que subraya el profesorado experimentado como beneficio para su desarrollo profesional académico.

- Respecto a los obstáculos son la falta de tiempo (Díaz, 2000) y el compaginar multitud de tareas de diferente naturaleza (Lucas, 2001) lo que subrayan los y las participantes.

- El profesorado novel se siente satisfecho con la mentoría porque les ayuda a dibujar el itinerario para desarrollar la competencia académica y, de su mano, lograr las acreditaciones por parte de las agencias externas, al hilo de lo que diferentes autores/as comentan (Harvey et al., 2016; Mullen, 2012). El profesorado novel subraya, además, que el hecho de sentirse acompañado les reconforta (Smith e Ingersoll, 2004) y les satisface profesionalmente (Malderez y Wedell, 2007). El profesorado mentor, por su parte, subraya el acompañamiento que desde la institución se lleva a cabo de guía y respaldo a su tarea.

La repercusión que la mentoría inserta en el programa formativo iRAKER ha tenido en el desarrollo docente, investigador y de gestión del profesorado novel a nivel individual e institucional destaca las siguientes cuestiones:

- El ejercicio de construcción del PAP individual marca y guía el proceso formativo acompasado por la mentoría, a modo de auto evaluación de necesidades (Díaz, 2000), ayudando a que el/la novel tome conciencia de las dimensiones y aspectos que configuran su desarrollo profesional como académico/a (Conde y Martín, 2016). Es en el ámbito docente en el que mayor efecto ha tenido por introducir cambios en sus prácticas incidiendo en el ámbito en el que la literatura subraya que la formación de los y las noveles es escasa (Fernández, 2003; Sánchez y Mayor, 2006).

- La mentoría ha inducido a que el profesorado novel asuma el reto de responsabilizarse de cargos académicos que, hasta el momento, creía que no le competían, así como en el ámbito personal relativo a la actitud hacia la formación continua de la que han tomado conciencia (Conde y Martín, 2016).

- El profesorado experimentado al asumir el rol de mentor/a ha aprendido a través de esta relación (Orland-Barak, 2016) en torno a su propia labor académica; a ser conscientes del desarrollo de habilidades de análisis y reflexión sobre su práctica.

- La mentoría propicia que el profesorado novel adquiera compromisos (Falzarano y Zipp, 2012) respecto a la gestión universitaria y del valor de la docencia, no sólo como parte de sus funciones, sino como contexto de innovación e investigación (Paricio, Fernández y Fernández, 2019). Por lo tanto, la mentoría impulsa el 
compromiso del profesorado novel con actividades y responsabilidades institucionales que inciden en la mejora y calidad universitaria.

Pautas para la transferencia:

El programa formativo diseñado al amparo de la estrategia de la mentoría ha de ser una apuesta estratégica de cada universidad para formar al profesorado que asume el testigo de trabajar, impulsar, desarrollar y liderar dicha institución. Para ello es importante acompasar la trazabilidad y sostenibilidad del programa formativo con los intereses de la institución universitaria. Para ello se debe poner el acento en:

- Definir criterios para la inclusión en procesos de mentoría tanto del profesorado novel (perfil académico y situación actual para las acreditaciones) como experimentado (trayectoria y bagaje en los diferentes ámbitos del desarrollo académico universitario). $\mathrm{Al}$ objeto de clarificar a qué intereses responde el programa formativo asentado en la estrategia de la mentoría.

- Trabajar estrechamente con las direcciones de los departamentos para que la inserción de los y las noveles en un proceso de mentoría cubra realmente, no sólo las necesidades formativas del docente, sino también las de la propia institución.

- Diseñar itinerarios individualizados competenciales que requieran un ejercicio de introspección a través del cual analizar objetivamente el punto de partida de cada novel y pautar los hitos para llegar a dichas metas en un período de tiempo determinado.

- Clarificar inicialmente a través de los itinerarios individuales de desarrollo académico cual/es de los ámbitos de actuación es el más deficitario (docencia, investigación...) para que la estrategia de la mentoría incida más estrechamente en él/ellos.

- Introducir en la mentoría objetivos vinculados con la producción científica (área de conocimiento y enseñanza-aprendizaje del área) y, la visibilización de las actividades de responsabilidad y compromiso social.

- Alimentar el repositorio de personas mentoras de las diferentes áreas del conocimiento y los distintos departamentos. Es recomendable y deseable que en cada uno de los departamentos existan una o varias personas dispuestas a realizar esta función. Por lo tanto, es importante dimensionar adecuadamente la expansión de la mentoría contando con el número de personas mentoras formadas para que desempeñen su papel.

- Provocar en el profesorado mentor que el análisis y la reflexión sobre la práctica académica sea una constante a lo largo del proceso formativo. Para ello el programa o apoyo institucional les ha de acompañar y hacer consciente de esta necesidad.

- Ofrecer una formación acreditativa como mentores/as al objeto de que sea una verdadera labor reflexiva y comprometida con la cultura universitaria y construcción de la identidad académica.

- Procurar que las personas mentoras tengan, al menos, la competencia de investigación y docencia acreditadas por organismos competentes. Por una parte, por el modelo que representan para el profesorado novel (inclusión de tareas de índole distinta, pero con la misma importancia para la academia) y, por otra, por el peligro que puede existir al descompensar las tareas de los diferentes ámbitos de actuación del PDI. 
- Introducir en el proceso de mentoría dispositivos que garanticen el seguimiento del acompañamiento del profesorado novel: permanente comunicación a través de foros con el profesorado mentor, al objeto de establecer una comunicación fluida que retroalimente el proceso.

- Crear mecanismos que garanticen el acompañamiento desde la institución al profesorado mentor, de tal manera que se les facilite materiales, espacios virtuales para preguntar y resolver dudas, espacios colaborativos para la discusión, entrevistas pautadas, entre otros.

\section{REFERENCIAS BIBLIOGRÁFICAS}

Aguilar, S. y Barroso, J. (2015). La triangulación de datos como estrategia en investigación educativa. Revista de Métodos y Educación, (47), 73-88.

Alkorta, I. (2012). IKD, un modelo para la educación superior en el siglo XXI. Fabrikart, (10), 14-25.

Álvarez, V., Romero, S., Gil, J., Rodríguez, J., Clares, J., Asensio, I., del Frago. R., García, B., García, M., González, B., Guardia, S., Ibarra, M., López, R., Rodríguez, G. y Salmerón, P. (2011). Necesidades de formación del profesorado universitario para la adaptación de su docencia al Espacio Europeo de Educación Superior. Revista Electrónica de Investigación y Evaluación Educativa, 17(1).

Allen, T.D., Johnson, H. M., Xu, X., Biga, A., Biga, A. \& Ottinot, R. C. (2009). Mentoring and Protégé Narcissistic Entitlement. Journal of career development, 31. https://doi. org/10.1177/0894845308327735

Allen, T.D. y Eby, L.T. (2010). The Blackwell Handbook of Mentoring. A Multiple Perspectives Approach. Australia: Blackwell publishing.

Anafarta, A. y Apaydin, C. (2016). The Effect of Faculty Mentoring on Career Success and Career Satisfaction. International Education Studies, 9(6), 22-31. https://doi.org/10.5539/ies.v9n6p22

Andreucci, P. (2018). La Supervisión de Prácticas Docentes: Una Deuda Pendiente de la Formación Inicial de Profesores. Estudios Pedagógicos, 39(1), 7-26. https://doi.org/10.4067/S071807052013000100001.

Arandia, M. y Rekalde, I. (2014). La formación de equipos de coordinación para el desarrollo curricular de los grados: el programa ehundu de la UPV/EHU. Revista de docencia universitaria, 12(4), 91-114.

Araneda, A. (2006). La triangulación como técnica de cientificidad en investigación cualitativa pedagógica y educacional. Revista de Estudios y Experiencias en Educación, 5(10), 11-38.

Arregi, N., Barañano, E., Eraña, J., Jorge, S., Juaristi, A., Larrinaga, A. y Mesanza, S. (2008). Reglamento de la UPV/EHU para la protección de carácter personal. Bilbao: Servicio Editorial de la Universidad del País Vasco.

Ballesteros, M. y Nubiola, J. (2013). Los mentores, una figura esencial de la Universidad. Propuesta para la formación de mentores. Recuperado de http://som.esbrina.eu/aprender/docs/6/ BallesterosPanizoMapiNubiolaJaime.pdf.

Beltrián, E., Galitón, N., García, N., Jové, G. y Macarulla, M. (2013). La triangulación múltiple como estrategia metodológica. Revista Iberoamericana sobre Calidad, Eficacia y Cambio en Educación, 11(4), 5-24.

Bergevin, Ch. \& Martineau, S. (2007). Le mentorat. Laboratoire d'analyse de l'insertion professionnelle en enseignement. Montreal: Université du Québec.

Blaxter, L., Hughes, C. y Tight, M. (2008). Cómo se investiga. Graó: Barcelona.

Boyer, E. (1990). Scholarship reconsidered: priorities of the professorate. The Carnegie Foundation for the Advancement of Teaching. New Jersey: Princeton University Press. 
Bozu, Z. (2010). El profesorado universitario novel: Estudio teórico de su proceso de inducción o socialización profesional. Revista Electrónica de Investigación y Docencia, 3, 55-72.

Braun, V. \& Clarke, V. (2006). Using thematic analysis in psychology. Qualitative Research in Psychology, 3(2), 77-101.

Callejo, J. (2001). El grupo de discusión: introducción a una práctica de investigación. Barcelona: Ariel.

Cano, E. (2016). Factores Favorecedores y Obstaculizadores de la Transferencia de la Formación del Profesorado en Educación Superior. REICE. Revista Iberoamericana sobre Calidad, Eficacia y Cambio en Educación, 14(2), 133-150. https://doi.org/10.15366/reice2016.14.2.008

Casas, J., Repullo, J. R. y Donado, J. (2003). La encuesta como técnica de investigación. Elaboración de cuestionarios y tratamiento estadístico de los datos. Aten Primaria, 31(8), 527-538.

Cebrián, M. (2011). La supervisión con e-Portafolios y su impacto en las reflexiones de los estudiantes en el Practicum. Estudio de caso. Revista de Educación, 354, 183-208.

Cherian, F. (2007). Learning to teach: Teacher candidates reflect on the relational, conceptual and contextual influences of responsive mentorship. Canadian Journal of Education, 30(1), 25-46.

Collins, A., Hawkins, J. \& Fredriksen, J. R. (1991). Three Different Views of Students: The Role of Technology in Assessing Student Performance. Journal of the Learning Sciences, 3(2), 205-217. https://doi.org/10.1207/s15327809j1s0302_3

Comité de Ética para las Investigaciones relacionadas con Seres Humanos de la UPV/EHU (CEISH, 2019). https://www.ehu.eus/es/web/ceid/ceish/comite

Conde, J. y Martín, A. (2016). Potencialidades y necesidades de mejora en la formación de profesores noveles universitarios. REIDE, 18(1), 140-152.

Cullingford, C. (2016). Mentoring in Education. An International Perspective. New York: Routledge.

Díaz, W. (2018). Formación del profesorado universitario, evaluación de la actividad docente, recursos y promoción profesional. Estudios Pedagógicos, 42(1), 65-85. https://doi.org/10.4067/ S0718-07052016000100005

Díaz, F. (2000). Mentoring: Una relación de confianza. Capital Humano, 137, 72-74.

Drew, L. (2004). The experience of teaching creative practices: conceptions and approaches to teaching in the community of practice dimension. Recuperado de http://ualresearchonline.arts. ac.uk/644/1/cltad_ped_res_pap_linda_drew.pdf

Eirín, R., García, H. M. y Montero, L. (2009). Profesores principiantes e iniciación profesional. Estudio exploratorio. Revista de currículum y formación del profesorado, 13(1), 101-115.

ENQA -European Association for Quality Assurance in Higher Education- (2015). Standards and guidelines for quality assurance in the Higher Education Area. Bruselas: European Union.

Falzarano, M. y Zipp, G. P. (2012). Perceptions of Mentoring of Full-Time Occupational Therapy Faculty in the United States. Occupational Therapy International, 19(3), 117-126. https://doi. org/10.1002/oti.1326

Feixas, M., Duran, M., Fernández, I., Fernández, A., Garcia, M. J., Márquez, M. D., Pineda, P., Quesada, C., Sabaté, S., Tomás, M., Zellweger F. y Lagos, P. (2013). ¿Cómo medir la transferencia de la formación en Educación Superior?: el cuestionario de factores de transferencia. Revista de Docencia Universitaria, 11(3), 219-248.

Fernández, A. (2003). Formación pedagógica y desarrollo profesional de los profesores de universidad: Análisis de las diferentes estrategias. Revista de Educación, 331, 171-197.

Fernández, C., González, Ma . R., y Belando, Mª . R. (2017). Mentoría pedagógica para profesorado universitario novel: estado de la cuestión y análisis de buenas prácticas. Estudios sobre educación, 33, 49-75.

Fernández, A. (2008). La formación inicial del profesorado universitario: el título de Especialista Universitario en Pedagogía Universitaria de la Universidad Politécnica de Valencia. Revista Interuniversitaria de Formación del Profesorado, 63(22), 161-187.

Fernández, I., Guisasola, G., Garmendia, M., Alkorta, I. y Medinabeitia, A. (2013) ¿Puede la 
formación tener efectos globales en la universidad? Desarrollo docente, metodologías activas y curriculum híbrido. Infancia y aprendizaje, 36(3), 387-400.

Fernández, I. y Palomares, T. (2011). ¿Cómo desarrollar un currículum universitario en la sociedad del conocimiento? IKD, un modelo de desarrollo en la Universidad del País Vasco. En N. Balluerka e I. Alkorta (Eds.). Desarrollo curricular de las nuevas titulaciones de grado (pp.122). Bilbao: Servicio Editorial de la Universidad del País Vasco.

Fernández, I. y Rekalde, I. (2011). Una universidad que aprende: innovación y cambio educativo en la UPV/EHU. Bilbao: publicaciones de la UPV/EHU.

Fletcher, S. J. y Mullen, C. A. (2012). Mentoring and Coaching in Education. California: Sage Handbooks.

Flick, U. (2011). Introducing research methodology. A beginner's guide to doing a research project. London: Sage.

Gibbs, G. \& Coffey, M. (2004). The Impact Of Training Of University Teachers on their Teaching Skills, their Approach to Teaching and the Approach to Learning of their Students. Active learning in Higher education, 5(1), 87-100. https://doi.org/10.1177/1469787404040463

Gijón, J. y Crisol, E. (2012). La internacionalización de la Educación Superior. El caso del Espacio Europeo de Educación Superior. REDU, 10(1), 389-414.

González, A. I., Araneda, N., Hernández, J. y Lorca, J. (2005). Inducción profesional docente. Estudios pedagógicos, $X X X I(1), 51-62$.

Grundy, S. (1991). Producto y praxis del currículum. Madrid: Morata.

Harvey, M., Ambler, T. \& Cahir, J. (2017). Spectrum Approach to Mentoring: An evidence-based approach to mentoring for academics working in higher education. Teacher Development, 21(1), 160-174. https://doi.org/10.1080/13664530.2016.1210537

Hativa, N. (2000). Teaching for Effective Learning in Higher Education. Dordrecht: Kluwer Academic Publishers.

Herrera, L., Fernández, A. M., Caballero, K. y Trujillo, J. M. (2011). Competencias docentes del profesorado novel participante en un proyecto de mentorización. Implicaciones para el desarrollo profesional universitario. Revista de currículum y formación del profesorado, 15(3), 213-241.

Herreras, E. (2007). El profesorado de educación superior. Formación para la excelencia. Revista de Educación Superior, 144(4), 139-141.

Hobson, A., Ashby, P. Malderez, A. \& Tomlinson, P. (2009). Mentoring beginning teachers: What we know and what we don't. Teaching and Teacher Education, 25(1), 207-216.

Infesta, G., Vicente, A. y Cohen, A. (2012). Reflexiones en torno al trabajo con grupos de discusión en Ciencias sociales. Intersticio. Revista Sociológica de Pensamiento Crítico, 6(1), 233-244.

Janesick, V. (2000). The Choreography of Qualitative Research Design: Minuets, Improvisations, and Crystallization. In N.K. Denzin \& Y. S. Lincoln (Eds.). The Handbook of Qualitative Research (pp. 379-400). Thousand Oaks, California: Sage Pub.

Jiménez, D., González, J. y Tornel, M. (2019). Formación del profesorado universitario en metodologías y su incidencia en el aula. Estudios Pedagógicos, 44(3), 157-172. https://doi. org/10.4067/S0718-07052018000300157

Jordi, M. y Macías, B. (2014). La entrevista en profundidad como recurso pedagógico en los estudios de Trabajo Social y Educación Social. Potencialidades y retos para el aprendizaje teóricopráctico. Revista de Investigación en Educación, 12(1), 2014, 105-111.

Kirkpatrick, D. y Kirkpatrick. J. (2000). Evaluación de acciones formativas: los cuatro niveles. Barcelona: Epise.

Knight, J. (2006). Internationalization of higher education: New directions, new challenges. The 2005 IAU global survey report. Paris: International Association of Universities.

Laverick, D. M. (2016). Mentoring Processes in Higher Education. USA: Springer Briefs in Education.

Light, G., Calkins, S., Luna, M. \& Drane, D. (2009). Assessing the Impact of a Year-Long Faculty 
Development Program on Faculty Approaches to Teaching. International Journal of Teaching and Learning in Higher Education, 20(2), 168-181.

Lobato, C. y Guerra, N. (2016). La tutoría en la educación superior en Iberoamérica: Avances y desafíos, Educar, 52(2), 379-398.

López, J. I. (2011). Un giro copernicano en la enseñanza universitaria: Formación por competencias. Revista de Educación, (356), 279-301.

Lucas, K. F. (2001). The social construction of mentoring roles. Mentoring and Tutoring, 9(1), 23-47.

Lukas, J. F. y Santiago, K. (2009). Evaluación educativa. Madrid: Alianza.

Madinabeitia, A. y Fernández, I. (2017). El desarrollo docente del profesorado universitario: Análisis y sistematización del concepto. Revista Interuniversitaria de Teoría de la Educación, 29(2), $87-108$.

Madriz, G. (2008). La entrevista en profundidad. Dos entre-vistas y una mirada. Revista ensayo y error, 17(35), 137-152.

Malderez, A. y Wedell, M. (2007). Teaching Teachers: Processes and Practices. London: Continuum.

Marentic, B. (2009). Improving the quality of teaching and learning in higher education through supporting professional development of teaching staff. Napredak, 150(3-4), 341-359.

Margalef, L. y Álvarez, J. M. (2005). La formación del profesorado universitario para la innovación en el marco de la integración del Espacio Europeo de Educación Superior. Revista de Educación, $337,51-70$.

Mas, O. (2011). El profesor universitario: sus competencias y formación. Revista de currículum y formación del profesorado, 15(3), 196-211.

Orbea-Aizpitarte, G., Cruz-Iglesias, E. y Rekalde-Rodríguez, I. (2019). ¿Cómo puede el portafolios del alumnado ayudar a mejorar las prácticas docentes? Revista Digital de Investigación en Docencia Univesitaria, 13(1), 17-37. https://doi.org/10.19083/ridu.2019.769

Ojeda, E. (2007). Interdisciplinariedad académica: en busca del nodo perdido. Cuadernos de pedagogía Universitaria, 4(8), 13-18. Recuperado de http://cuaderno.pucmm.edu.do/index.php/ cuadernodepedagogia/article/view/69

Orland-Barak, L. (2016). Mentoring. In J. Loughran \& M. L. Hamilton (Eds.). International Handbook of Teacher Education (pp.105-141). Singapore: Springer Publications.

Palomares, A. (2011). El modelo docente universitario y el uso de nuevas metodologías en la enseñanza, aprendizaje y evaluación. Revista de Educación, 355, 591-604. http://doi.org/104438/1988-592X-RE-2011-355-038.

Paricio, J., Fernández, A. y Fernández, I. (Eds.). (2019). Cartografía de la buena docencia universitaria. Un marco para el desarrollo del profesorado basado en la investigación. Madrid: Narcea.

Patton, M. Q. (2002). Two Decades of Developments in Qualitative Inquiry: A Personal, Experiential Perspective. Qualitative Social Work, 1(3), 261-283. https://doi.org/10.1177/14733250020010 03636

Pineda, P. (2000). Evaluación del impacto de la formación en las organizaciones. Educar, (27), 119133.

Postareff, L., Lindblom-Ylänne, S. \& Nevgi, A. (2008). A follow-up study of the effect of pedagogical training on teaching in higher education. Higher Education, 56(1), 29-43. Recuperado de http:// link.springer.com/article/10.1007/s10734-007-9087-z

Rekalde, I. y Guerra, N. (2018). El mentoring. Una estrategia de acompañamiento en el desarrollo de la competencia académica del profesorado universitario. Evaluación de la Calidad de la Investigación y de la Educación Superior. Libro de resúmenes XV FECIES (p. 504). Granada: Asociación Española de Psicología Conductual.

Rippon, J. H. \& Martin, M. (2006). What makes a good induction supporter? Teaching and Teacher Education, 22(1), 684-702.

Sánchez, M. y Mayor, C. (2006). Los jóvenes profesores universitarios y su formación pedagógica. Claves y controversias. Revista de Educación, 339, 923-946. 
Sánchez, P., Chiva, I. y Perales, M. J. (2015). Experiencia en la formación docente a través de la mentorización. Revista Electrónica de Investigación Educativa, 17(1), 33-54. Recuperado de http://redie.uabc.mx/vol17no1/contenido-schezchivap.html

Smith, T. M. \& Ingersoll, R. M. (2004). What are the effects on induction and mentoring on beginning teacher turnover? American Educational Research Journal, 41(3), 681-714.

Shulman, L. (1999). Taking Learning Seriously, Change, 31(4), 10-17. doi: http://doi. org/10.1080/00091389909602695

St-Jean, E. y Audet, J. (2013). The Effect of Mentor Intervention Style in Novice Entrepreneur Mentoring Relationships. Mentoring and Tutoring 21(1). https://doi.org/10.1080/13611267.20 13.784061

Stake, R. E. (1998). Investigación con estudios de casos. Madrid: Morata.

Taylor, S. J. y Bogdan, R. (1996). Introducción a los métodos cualitativos de investigación. Paidos: Barcelona.

Tójar, J. C. (2001). Planificar la investigación educativa: una propuesta integrada. Fundec.

Trigwell, K. \& Prosser, M. (2004). Development and use of the approaches to teaching inventory. Educational Psychology Review, 16(4), 409-424.

Vázquez, R. y Angulo, F. (2003). Introducción a los estudios de casos. Los primeros contactos con la investigación etnográfica. Málaga: Aljibe.

Vélaz de Medrano, C. (2009). Competencias del profesor-mentor para el acompañamiento al profesorado principiante. Revista de currículum y formación del profesorado, 13(1), 210-229.

Vonk, J.H.C. (1996). Conceptualizing the mentoring of beginning teachers. Speeches/Meeting Papers. Recuperado de $:$ https://eric.ed.gov/?q=\%22Conceptualizing+the+mentoring+o f+beginning+ teachers $\% 22+$ Vonk\&id=ED400241

Zabalza, M. A. (2003). Las competencias docentes del profesorado universitario: calidad y desarrollo profesional. Madrid: Narcea. 
